\title{
STATCOM Controller Performance Enhancement and Optimization Technique
}

\author{
Tariq Masood ${ }^{1}$, Nasir Jameel ${ }^{2}$, Suhail Aftab Qureshi ${ }^{3}$, Muhammad Tajammal $^{4}$ \\ Samer Karim Haider ${ }^{5}$, Ghulam Hashmi ${ }^{6}$ \\ ${ }^{1}$ Asset Integrity Department, Dukhan Operations, Qatar Petroleum, Email: T.Masood.Dr@ieee.org \\ ${ }^{2}$ Maintenance Department Schlumberger Surface Maintenance Australia: Email:nasirjameel130@gmail.com \\ ${ }^{3}$ University of Engineering and Technology, Lahore Pakistan: Email: tiger suhail@hotmail.com \\ ${ }^{4}$ University of Hail, Hail, Saudi Arabia: Email: mt.chughtai@uoh.edu.sa \\ ${ }^{5}$ Maintenance Department QAFAC Doha, Qatar: Email: samerk@qafac.com.qa \\ ${ }^{6}$ Power System Engineering Department Saudi Aramco, Dhahran, Saudi Arabia: Ghulam.hashmi@,Aramco.com
}

\begin{abstract}
STATCOM controller is a novel power transmission governing device which provides complete dynamic control management of Electrical power transmission system network's at various operating parameters. This research investigation of a STATCOM device offers a progressive governance for the control and operation of a STATCOM Controller which has been coupled in the GCC interconnection between the United Arab Emirates and Kuwait. The principal aim of this investigative work is to reconnoitre the encounters of power transmission network and operational control. The investigation will also inspect the positive impact of employing a STATCOM within progressive control algorithm to design and regulate three operating boundaries of compensation factors for transmission network. The investigation results denote that the STATCOM is a robust contestant to improve the operations for electrical power transmission systems.
\end{abstract}

\section{Key words}

Voltage Control, Reactive Power, Stability, Damping

\section{Introduction}

The progressive control of a STATCOM device is presented, which has discovered a vigorous and irrepressible positive influence on improving the voltage quality and stability of interconnection at the GCC power grid between United Arab Emirates and Kuwait.

The FACTS Controller selection process has been investigated by using Wideband Delphi Technique [1], [2], [3].

In this study 500Mvar $400 \mathrm{kV}$ STATCOM suggested to deliver dynamic control of the power transmission network. In order to adjust the STATCOM response, three operating boundaries have been configured and simulated. The simulated model showed promising results by addressing the following factors as attributed:-(1).Power- flow is not optimally coordinated and adjusted. (2). The reactive-power is reserved to be well controlled and managed. (3). Electrical power network operation's stability is not controlled completely. Therefore, the investigation results denoted that the STATCOM is a robust contestant to improve electrical power transmission system operations. The technology options on the basis of following benefits can easily be determined and witnessed as listed below [2, 3]:-

1. Augmented Electrical power-transfer capability.

2. Achievement of enhanced electrical power system network reliability.

3. Enhanced Voltage and Angle stabilities in the GCC power network.

Figure 1 shows the determined power which is commutable on the bases of each GCC country's rigor requirements. This investigative work supplies the basis to authenticate the aspects of power system network on the GCC network as mentioned below:-.

- Examines theoretically the transferability of maximum electrical power.

- Examines improvement in voltage if STATCOM is employed at the midpoint.

- Examines how much real power is needed for sharing purposes if midpoint voltage is maintained at a value of $0.98 \mathrm{pu}$.

\section{GCC Electrical Power System -Network}

The GCC power system network was designed and implemented into eight strategic control boundaries in order to meet rigorous operational requirements as per the defined boundaries of each participating GCC Country. 


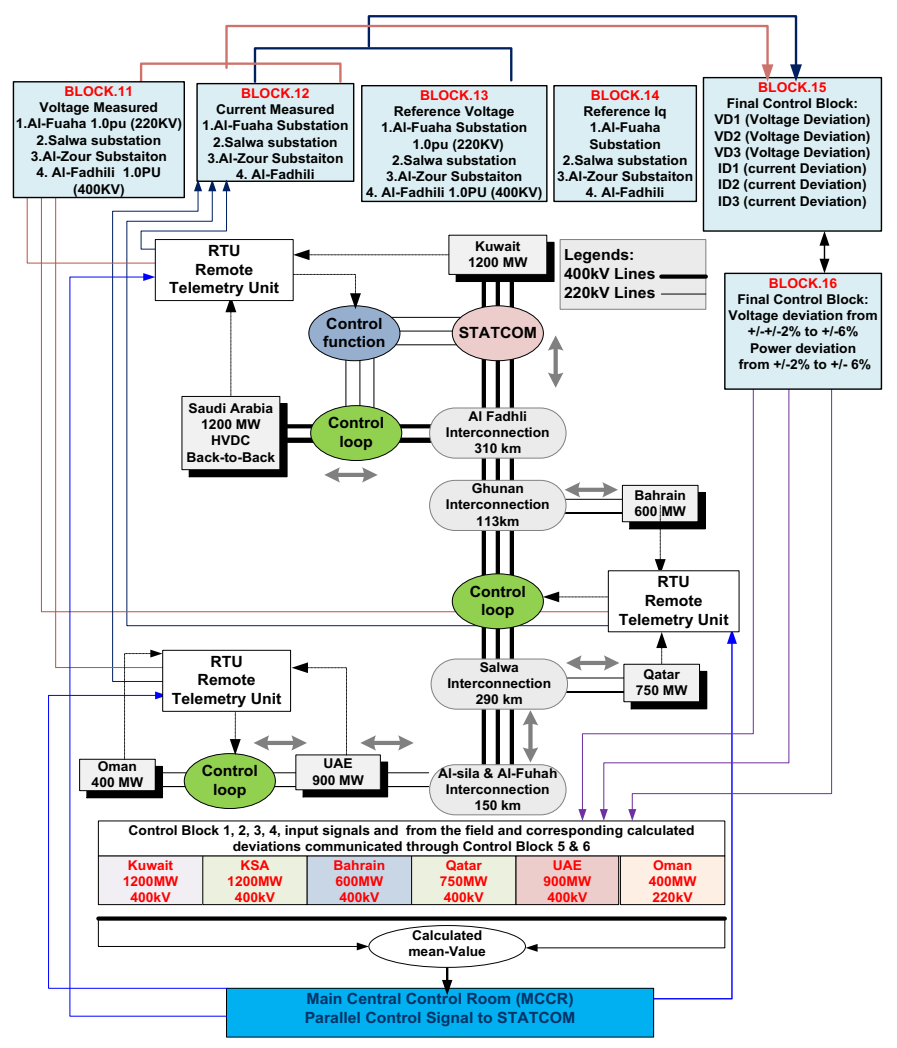

Figure 1: GCC countries current power sharing operations.

\section{STATCOM Calculated Model}

\section{A. Electrical Power Delivery Without Compensation}

The GCC interconnection power transmission network in between United-Arab-Emirates and Kuwait have the subsequent characteristic technical values:

The power transmission line operates at a voltage $(\mathrm{V})$ of $400 \mathrm{kV}$ from Kuwait to the United-Arab-Emirates; having frequency $(\mathrm{F})$ of $50 \mathrm{~Hz}$; covering the distance from Kuwait to the United-Arab-Emirates which is $850 \mathrm{kms}$; and contains line inductance of $0.89 \mathrm{mH}$ per $\mathrm{km}$; and capacitance (C) of $8.7 \times 10^{-9} \mathrm{~F} / \mathrm{km}$. The midpoint voltage on the GCC power grid needed to be regulated at $0.9812 \mathrm{pu}$. Therefore, the transmission line's total impedance from Kuwait to United-Arab-Emirates is computed by using equation (1) and $\theta$ Equations (2) and (3), as stated below: The power transmission line impedance was computed equal as $327 \Omega$ and a surge impedance of $493 \mathrm{Ohms}$, as given below:

$Z_{a}=\sqrt{\frac{l(n H)}{c\left(\frac{C}{V}\right)}}$

$\operatorname{Beta}(\beta)=\omega \sqrt{(l) \times(c)}=0.047 \mathrm{Deg} / \mathrm{km}$

$\theta=\beta \times d=39.7^{\circ}$

$P_{\text {max }}=\frac{V^{2}}{Z_{a} \times \operatorname{Sin} \theta}=\frac{400^{2}}{327 \times \operatorname{Sin} 39.7^{\circ}}=771 \mathrm{MW}$

Equation (4) shows concentrated theoretical electrical power that can be dispensed without any constraints from Kuwait to the United-Arab-Emirates. Figure 2 signposts the correlation of $\mathrm{P}, \mathrm{S}$, and $\mathrm{Q}$ in order to deliver 771.81
MW in between United-Arab-Emirates and Kuwait. The power factor will be in leading if $\mathrm{Q}$ bears a negative value, on contrary it will lag when $\mathrm{Q}$ has a positive value.

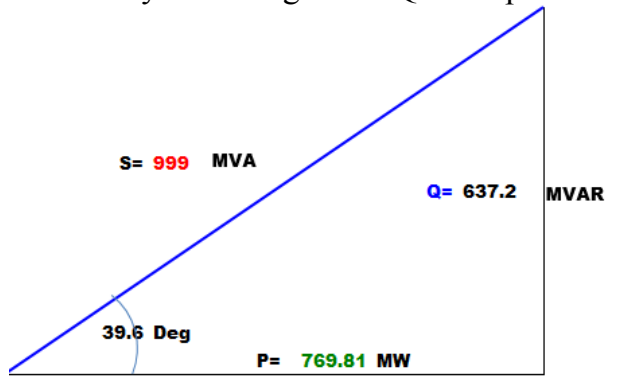

Figure 2: GCC power grid power triangle.

Set of Equations are discussed in the paper as Equation (5) denotes the way to calculate the shunt compensation factor and its three operating boundaries [5],[6].

$$
\left\{\begin{array}{c}
K_{\text {Shunt_Maximum }}=\frac{B_{c 1} Z_{a} \times \tan \frac{\theta}{2}}{X_{C} \times 2} \\
K_{\text {Shunt_Medium }}=\frac{B_{c 2} Z_{a} \times \tan \frac{\theta}{2}}{X_{C} \times 2} \\
K_{\text {Shunt_Minimum }}=\frac{B_{c 3} Z_{a} \times \tan \frac{\theta}{2}}{X_{C} \times 2} \\
B_{c}=B_{c 1}+B_{c 2}+B_{c 3}
\end{array}\right.
$$

$K_{\text {shunt }}=K_{\text {shunt_max }}+K_{\text {shunt_Medium }}+K_{\text {shunt_Minimum }}$ the $\mathrm{K}_{\text {shunt }}$ factor calculation indicates that $\mathrm{B}_{\mathrm{C}}$ is a very important factor, as discussed in Equation (5) and is responsible to vary the line reactance to support essential voltage level.

$$
B_{c}=\frac{\sqrt{3}-1}{\sqrt{3} X}
$$

Equation (6) is used to compute the $\mathrm{B}_{\mathrm{C}}$ factor. The " $\mathrm{X}$ " value can be varied from $0.1 \mathrm{pu}$ to $0.99 \mathrm{pu}$

Therefore, the $\mathrm{B}_{\mathrm{C}}$ factor can be computed to determine the power flow against the voltage drop at the receiving/sending ends of the power transmission network.

\section{B. Conventional Capacitor Control System}

In this study, the orthodox shunt-capacitor if employed at the midpoint to support the voltage has been calculated by using Equation (7). Equations (8) and (9) are referred to compute $\mathrm{P}$ and $\mathrm{P}_{\mathrm{n}}$ (surge impedance) $\mathrm{P}_{\max }$ is calculated as given below.

$$
\begin{aligned}
& V_{m}=\frac{V \times \cos \frac{\theta}{2}}{\cos \frac{\delta}{2}\left(1-K_{\text {shunt }}\right)} \\
& \cos \frac{\delta}{2}=0.981 \cos 39.7^{\circ}\left(1-\frac{327 \tan 39.7^{\circ}}{500 \times 2}\right) \\
& P_{n}=\frac{V^{2}}{Z_{a}}=491 \\
& P=\frac{V V_{m} \sin \frac{\delta}{2}}{Z_{a} \sin \frac{\theta}{2}}=\frac{1 \times 0.981 \times \sin 39.7^{\circ}}{\sin 19.9^{\circ}}
\end{aligned}
$$


$P_{\text {max }}=P \times P_{n}=1.681 \times 492=827 M W$

Equation (11) elucidates that a determined electrical power flow of $827 \mathrm{MW}$ can be supported in the $850-\mathrm{kms}-$ long power transmission line by employing a traditional shunt capacitor having a value of $8.7 \times 10^{-9} \mathrm{~F} / \mathrm{km}$.

\section{Voltage Support with STATCOM Controller}

The STATCOM controller has a tremendous ability to control-voltage in the dynamic mode of the power network by replacing the unproductive orthodox device (shuntcapacitors). Orthodox shunt capacitor having reactance value of 503 Ohms which was achieved $\delta=72^{\circ}$ and $\mathrm{V}_{\mathrm{m}}$ maintained at $0.981 \mathrm{pu}$. Equation (12) was denoted to determine the reactive power required for compensation at the midpoint in between United-Arab-Emirates and Kuwait [8].

$V_{m}=\frac{V \times \cos \frac{\delta}{2}}{\cos \frac{\theta}{2}}-\frac{I_{r} \times Z_{n} \times \tan \frac{\theta}{2}}{2}$

Equation (12) denotes how Vm voltage is calculated.

$I_{r}=2\left[\frac{\cos 39.61^{\circ}}{\cos 19.8^{\circ}}-\frac{0.98}{1}\right] \frac{1}{\tan 19.8^{\circ}}$

Equation (13) denotes the $I_{r}$ reactive current calculated during compensation.

$I_{r}=-V_{m} \times \frac{Z_{a}}{X_{c}}=0.98 \times 0.6=-0.638 p u$

Equation (14) denotes the formula to find Ir value to know how much power flow would had transmitted from Kuwait to United-Arab-Emirates, and Equation (15) denotes the determined power that can be recognized as given below.

$\bar{P}_{\max }=\frac{P}{P_{n}}=A \sin \delta+B \frac{\delta}{2}$

Where

$A=\frac{1}{\sin \theta}=1.5681$

$B=\frac{-I_{r}}{2 \times \cos \frac{\theta}{2}}=0.5031$

To support the midpoint transmission line voltage in between UAE and Kuwait, the 'P' values are computed as denoted in Equation (18).

$P=A \sin \delta+B \sin \frac{\delta}{2}$

The value of determined power is expressed by $P$ when $\frac{d P}{d \delta}=0$ as denoted in Equation (19)

$\frac{d P}{d \delta} \sin \delta_{p}=\cos \delta_{p}$

$\frac{d P}{d \delta} \sin \delta_{p}$ value is substituted from Equation (18)

$\frac{d P}{d \delta}=\frac{d}{d \delta}\left(A \sin \delta_{p}+B \sin \frac{\delta_{p}}{2}\right)$

Substituting $\frac{d P}{d \delta} \sin \delta_{p}$ value in Equation (20), Equation (21) is derived as given below
$0=\left(A \cos \delta_{p}+B \cos \frac{\delta_{p}}{2}\right)$

$0=A \cos \delta_{p}+B \cos \frac{\delta_{p}}{2}$

Substituting $\frac{d P}{d \delta} \sin \delta_{p}$ value in Equation (22), Equation (23) is derived as given below

$A \cos \delta_{p}+B \cos \frac{\delta_{p}}{2}=0$

$\delta_{p}$ at determined value, based on the determined power, can be produced as denoted in Equation (24)

$\frac{d P}{d \delta}=0=A \cos \delta_{p}+B \cos \frac{\delta_{p}}{2}$

Where

$\cos \delta_{p}=\cos \left(\frac{\delta_{p}}{2}+\frac{\delta_{p}}{2}\right)$

Equation (25) derives $\cos \delta_{p}$ to substitute with $x=\cos \frac{\delta}{2}$ Wherein $\cos (A+B)=\cos A \cos B-\sin A \cdot \sin B$

$(\mathrm{A}+\mathrm{B})$ is then substituted by $\frac{\delta_{p}}{2}+\frac{\delta_{p}}{2}$ in Equation (26) in order to derive Equation (27) as given below

$\cos \left(\frac{\delta_{p}}{2}+\frac{\delta_{p}}{2}\right)=\cos \frac{\delta_{p}}{2} \cdot \cos \frac{\delta_{p}}{2}-\sin \frac{\delta_{p}}{2} \cdot \sin \frac{\delta_{p}}{2}$

By simplifying Equation (27), Equation (28) is derived as:

$\cos \delta=\cos ^{2} \frac{\delta_{p}}{2}-\sin ^{2} \frac{\delta_{p}}{2}$

By substituting the values of $\cos \delta$ in Equation (24)

$0=A\left(\cos ^{2} \frac{\delta_{p}}{2}-\sin ^{2} \frac{\delta_{p}}{2}\right)+B \cos \frac{\delta_{p}}{2}$

Where

$\sin ^{2} \frac{\delta_{p}}{2}=\left(1-\cos ^{2} \frac{\delta_{p}}{2}\right)$

Substituting the value $\operatorname{Sin}^{2} \frac{\delta_{p}}{2}$ in Equation (29) and Equation (31) is derived as :

$0=A\left(\cos ^{2} \frac{\delta_{p}}{2}-\left(1-\cos ^{2} \frac{\delta_{p}}{2}\right)\right)+B \cos \frac{\delta_{p}}{2}$

By substituting $x=\cos \frac{\delta_{p}}{2}$ in Equation (31), Equation (32) is obtained as

$\left(2 x^{2}-1\right)+\frac{1}{2} B x=0$

simplifying equation 32 , yields

$2 A x^{2}-A+\frac{1}{2} B x=0$

Equation (33) can be used to organize the contents into quadratic form to reach:

$2 A x^{2}+\frac{1}{2} B x-A=0$

Equation (34) is converted into quadratic solution to get:

$\int(x)=\left(x+\frac{b}{2 a}\right)^{2}+\frac{4 a c-b^{2}}{4 a}$

Equation (35) defines the quadratic function as expressed in [9]
$\left(x+\frac{B}{2.4 . A}\right)^{2}+\frac{(4 \times 2 A \times-A)-\left(\frac{B}{2}\right)^{2}}{4 A}=0$

Rearranging Equation (36) gives

$\left(x+\frac{B}{8 A}\right)^{2}-\frac{8 A^{2}+\frac{B^{2}}{4}}{4 A}=0$ 
$\left(x+\frac{B}{8 A}\right)^{2}=\frac{8 A^{2}+\frac{B^{2}}{8}}{8 A}$

Equation (37) and (38) simplify the " $x$ "value to lead to

$x=-\frac{B}{8 A} \pm \frac{\sqrt{\frac{B^{2}}{4}+8 A^{2}}}{4 A}$

" $x$ " values are derived by substituting Equation (16) and (17) as follows:

$x=-\frac{0.5031}{8 \times 1.568} \pm \frac{\sqrt{8 \times 1.568^{2}+\frac{0.5031^{2}}{4}}}{4 \times 1.568}$

Equation (40) is used to compute the " $x$ " value. The calculated value of $\mathrm{x}=0.6681$ leads to maximum value of "P' at $\delta=96.21^{\circ}$

$$
P_{\max }=\hat{P} \max \times \frac{V^{2}}{Z a}
$$

Equation (41) proves to compute the $\mathrm{P}_{\max }$ value

$\hat{P} \max =A \sin 96.1^{\circ}+B \sin 39.6^{\circ}$

Equation (44) will elucidates the method to compute the value of $\hat{P} \max$ and to evaluate the maximum power dispensed in between the United-Arab-Emirates and Kuwait at persistent reactive power which has been computed using Equation (14)

$$
P_{\text {max }}=1.933 \times 491=949.1 \mathrm{MW}
$$

Equation (43) illustrates the determined power dispensed by employing STATCOM to improve midpoint voltage of the power network in between Kuwait and United-ArabEmirates. In conclusion, a STATCOM has adaptable capacity and capability to recuperate voltage and the stability margin of the power system against the orthodox shunt capacitor.

\section{STATCOM Distributed Control}

The distributed control technique is established and validated to vary the STATCOM controller response as per system requirements in line with power system impedance in three regimes of power system operations such as week, medium and strong system. Figure 4 denotes the first operative boundary for which lowest compensation is required from the STATCOM. If $\mathrm{X}_{\text {minimum }}=4.234 \Omega$ in terms of impedance and is referred to as strong power system network.

Figure 5 designates second operative boundary of compensator, if $X_{\text {medium }}=6.712 \Omega$ of impedance then the network is called medium strength's power system network. Figure 6 signifies third operative boundary of the compensator if the $X_{\text {maximum }}=10.2 \Omega$ of impedance, is known as weak power system network as detailed below.

- The compensator persisted in synchronous operative circumstances having $\mathrm{AC}$ power system at the mounted location and will compensate and address the bus voltage on time based contingencies.

- Due to nearby fault, if bus voltage is lost, it has the ability to evoke synchronism as soon as the fault is cleared.
- Distributed control has the capability to adjust the bus voltage deviation, certainly it leads to improve the transient stability.

- Distributed control topology has the capacity to damp out transients, to enhance system stability.

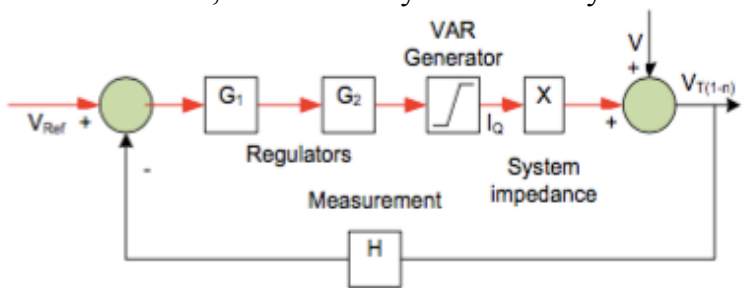

Figure 3: Feedback control loop transfer function

Figure 3 denotes the feedback control loop operative limits as computed below. The terminal voltage will be controlled against the power system voltage, if $\mathrm{V}_{\mathrm{Ref}}=0$ then small variation can be considered prudently.

$$
V_{\text {Terminal }}=\frac{1}{1+G_{1} G_{2} H X}=\frac{1}{1+G H X}
$$

Where

$$
\begin{aligned}
& G_{1}=\frac{1 / k}{1+T_{1} s} \\
& G_{2}=e^{-T_{S} d} \\
& G=G_{1} G_{2}=\frac{1 / k}{1+T_{1} s} e^{-T_{S} d} \\
& H=\frac{1}{1+T_{2} S}
\end{aligned}
$$

Table 1: STATCOM Controller Parameters

\begin{tabular}{|c|c|c|c|}
\hline Module & Parameter & Definition & Typical \\
\hline Measuring & $\mathrm{T}_{1}$ & For Time constant & $12 \mathrm{~ms}$ \\
\hline $\begin{array}{c}\text { System } \\
\text { Impedance }\end{array}$ & $\mathrm{X}_{\text {Min }}$ & $\begin{array}{c}\text { Reactive part of } \\
\text { the system }\end{array}$ & 4.213 \\
\hline $\begin{array}{c}\text { System } \\
\text { Impedance }\end{array}$ & $\mathrm{X}_{\text {Medium }}$ & $\begin{array}{c}\text { Reactive part of } \\
\text { the system }\end{array}$ & 6.213 \\
\hline $\begin{array}{c}\text { System } \\
\text { Impedance }\end{array}$ & $\mathrm{X}_{\text {High }}$ & $\begin{array}{c}\text { Reactive part of } \\
\text { the system }\end{array}$ & 10.21 \\
\hline Slope & $\mathrm{K}_{\mathrm{D}}$ & steady state error & 0.875 \\
\hline
\end{tabular}

Table No.1 denotes ultimate operative control parameters of the distributed Control technique as simulated in this study. This signifies that the PI (Proportional and Integral) control parameters can be varied within 15$55 \mathrm{~ms}$ to readjust STATCOM response. This depends on the var generator's lag at constant time (T1). Secondly, it also denotes T2 as time constant having a typical value of $6-25 \mathrm{~ms}$, for the amplitude measuring circuit.

Third control parameters is also known as Td which demonstrates Thyristor Control Reactor (TCR) response within a time span of $2.75 \mathrm{~ms}$ and Thyristor-Switched Capacitor (TSC) response within a time interval of 0.256 to $0.234 \mathrm{~ms}$. The parameter $\mathrm{X}$ is called the Im (imaginary) value and $\mathrm{Z}$ denotes the reactor part of electrical power system network's impedance. Fourth control block of the network is denoted by ' $\mathrm{k}$ ', this is also called the regulation slope (1-6) known as a laplace operator in the given equations [10].

\section{A. STATCOM Distributed Control to enhance transient stability}


The Distributed control system is capable to augment the electrical power system transients' stability. This enables the system to recover during major disturbances. These types of disturbances will transpire during severe faults on heavily loaded power transmission lines.

Based on electrical power system impedance the STATCOM response will be adjusted to compensate and overcome the small or large disturbances to maintain seamless operations. This controller will act within 0.012 second to address and increase the transient stability limit and to provide power oscillations damping robustly.

\section{B. STATCOM Distributed Control to enhance power oscillation damping}

During normal operations if the continuation of power oscillation in the power system network, due to dynamic behavior, necessitates implementation of shunt compensator. This readdresses the issue and the reactive power adjusts to meet essential reactive power requirement for the network. Inadequate reactive power may counteract decelerating or accelerating swings of the generator and midpoint voltage levels.

1. The generator oscillates and loads an increase in angle $\delta$ by $(\mathrm{d} \delta / \mathrm{dt}>0)$. When a gas turbine injects a mechanical power in to the generator rotor, this compensates excessive mechanical power which is essential for production of electrical power.

2. As soon as a Gas Turbine reduces its speed to decelerate the generator and load angle $\delta$ at a reduced level of $(\mathrm{d} \delta / \mathrm{dt}<0)$, it causes to reduce the electrical power to balance the induced mechanical power

3. Based on that the requisite VAR will be managed and readjusted to damp out the power system oscillations

4. The STATCOM response will increase the midpoint voltage as well as the transmitted power when $\mathrm{d} \delta / \mathrm{dt}$ $>0$, but it decreases immediately as soon as it bears the value of $\mathrm{d} \delta / \mathrm{dt}<0$.

5. The STATCOM response can be adjusted at three different levels (1) minimum (2) medium and (3) maximum compensation as rated in Figures 4,5 and 6.

In conclusion, this type of smart solution has capability and capacity to address time based contingencies likewise large oscillations and enhanced power operations stability.

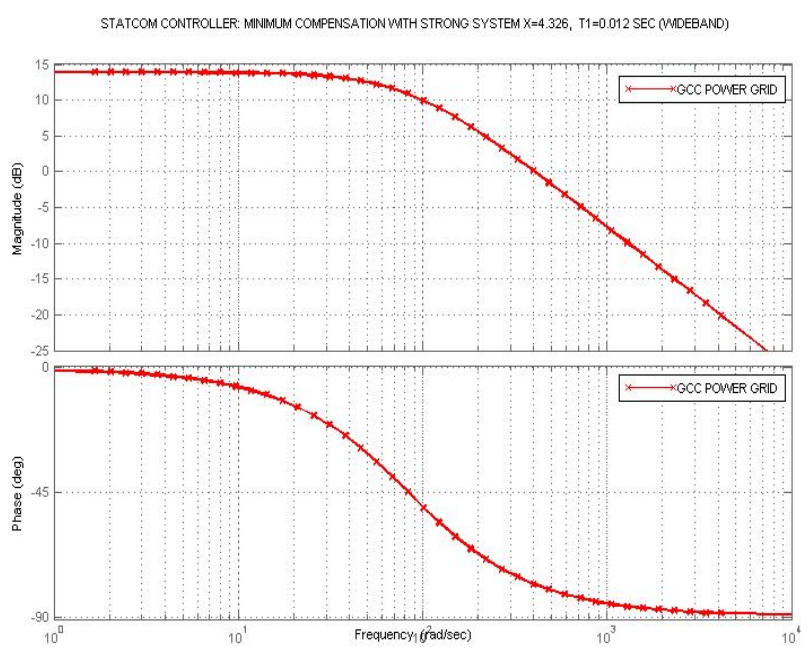

Figure 4:Bode plot when STATCOM regulates the voltage of the strong system

STATCOM CONTROLLR: MEDUMM COMPENSATION MTH MEDUMMSTFENOTH OF THE SYSTEM X=6 85, T1=0.012 SEC MMDEEAND)

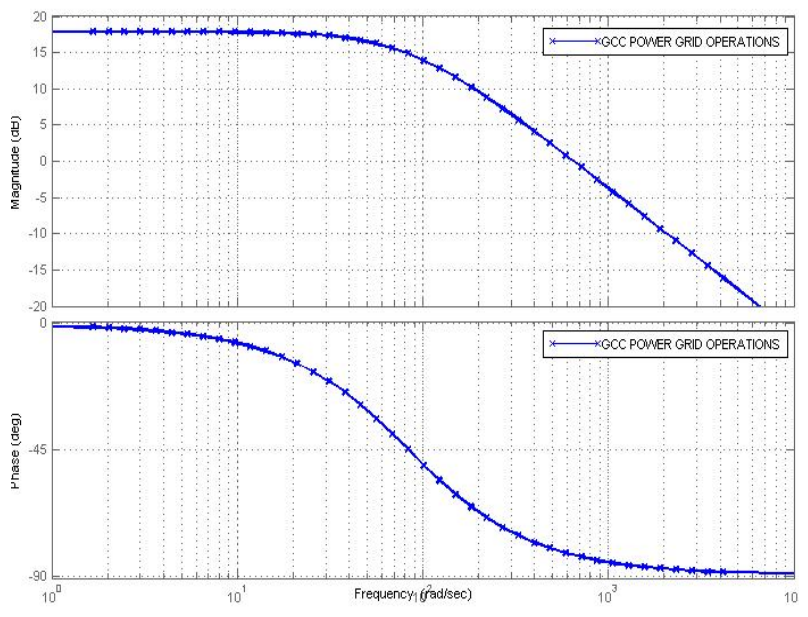

Figure 5: Bode plot when STATCOM regulates the voltage of the medium-strong system
STATCOM CONTROLLER: MAXMUM COMPENSATION MTH WEEK SYSTEM X $=10.51$, T1=0.012 SEC (MDEEAND)

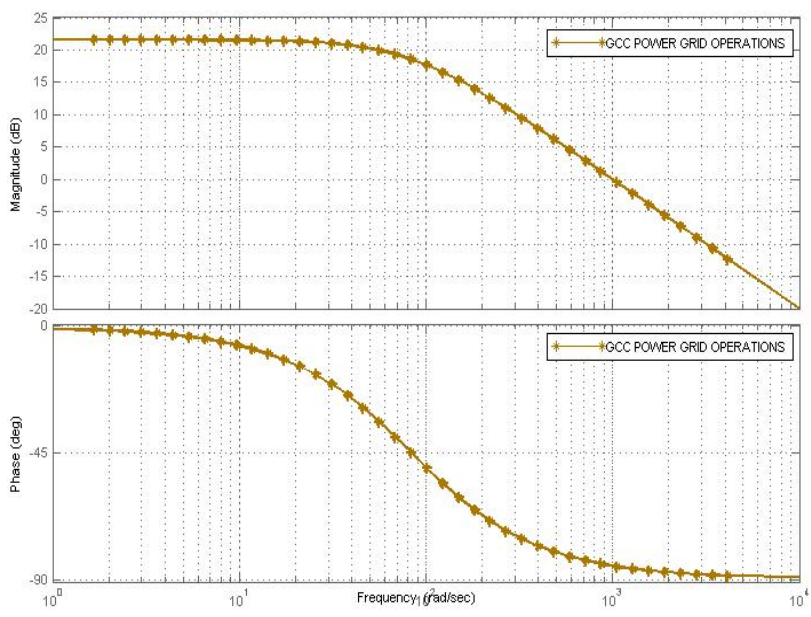

Figure 6: Bode plot when STATCOM regulates the voltage of the week system 


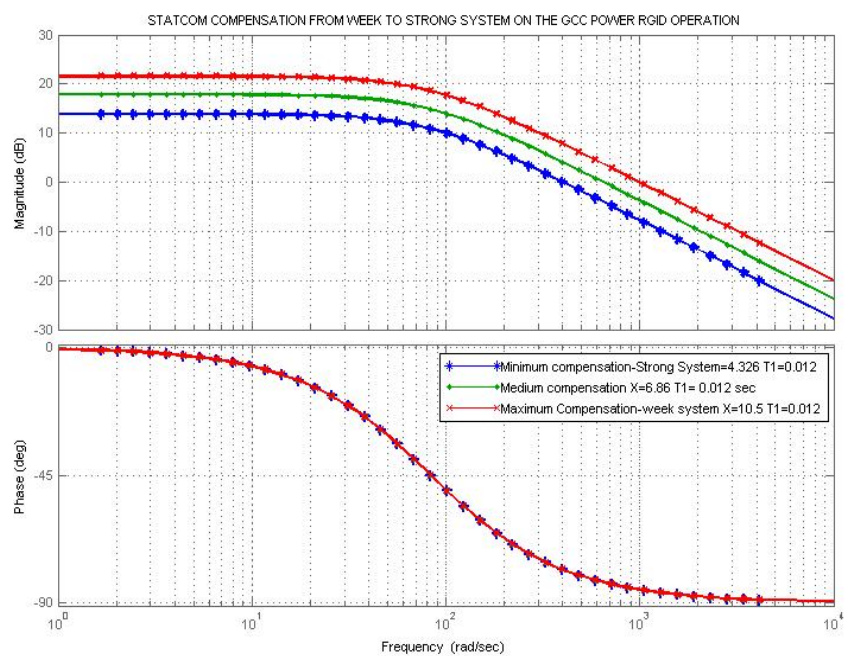

Figure 7: Bode plot when STATCOM regulates the voltage of week to strong power system

Table 2: Abbreviation and Synonyms

\begin{tabular}{|l|l|}
\hline Name & Abbreviations \\
\hline GCC & Gulf Cooperative Council \\
\hline Ir & Reactive current \\
\hline $\mathrm{Za}$ & Line Impedance \\
\hline $\mathrm{Vm}$ & Midpoint voltage \\
\hline $\mathrm{K}_{\text {shunt }}$ & shunt compensation factor in the network \\
\hline $\mathrm{Pn}$ & Surge Impedance \\
\hline
\end{tabular}

\section{Conclusions}

This study demonstrates the application of STATCOM and its proportional evaluation with traditional fixed shunt-capacitor compensation on the GCC power network. The study also proposed that the midpoint voltage of the network can be sustained in line with deviation and compensation factor. Based on the required suceptance was achieved to deliver the maximum real power as discussed. The STATCOM has a versatile ability to progress the voltage deviation if persistent in the network and augment the power system network stability margin on the GCC power grid with degree of precision.

\section{References}

[1]. Tariq Masood, Abdel Aty-Edris, S.A. Qureshi, Muhammad Tajammal, Murtaza Hashami, D.P. Kothari, Samer Karim Shah 'Smart grid operations and control challenge by implementing STATCOM tailored to optimize performance in between United Arab Emirates \& Kuwait at the GCC power grid published in the $13^{\text {th }}$ IET International Conference ACDC2017 at Midland Manchester, UK

[2]. Tariq Masood, R.K. Aggarwal, Nasser Al-Emadi, S.A. Qureshi, D.P.Kothari, 'Novel selection approach of a STATCOM Device Tailored and Optimized Engineer ing Configuration to Implement in Between United Arab Emirates and Kuwait", volume 3 Issue 3 March 2014, International Journal Automation and Power Engineering (www.ijape.com)

[3]. FU Rong, SUN Wanpen "Study on Application of STATCOM in Voltage Stability of Wind Farm
Incorporated System" $32^{\text {nd }}$ Chinese Control Conference July 26-26 2013

[4]. Piyushkumar M. Saradva "Application of DSTATCOM to Control Power Flow in Distribution Line" published in 2016

[5]. T. Tamilarasi, M. K. Elango, "Analysis of Impact on Rotor Angle Stability of DFIG Wind Turbines Employing STATCOM" published IEEE in 2016

[6]. Aditya P. Jayam, Nikhil K. Ardeshna, Badrul H. Chowdhury, "Application of STATCOM for Improved Reliability of Power Grid Containing a Wind Turbine" published IEEE in 2008

[7]. Shen Dong, Wang Zhonghong, J.Y.Chen, and Y.H.Song "Harmonics resonance phenomena in statecom and relationship to parameters selection of Passive components" IEEE Transaction on Power delivery, vol, 16, No. 1, January 2001.

[8]. Tariq Masood, R.A.J Khan "STATCOM and SVC Control Operations and Optimization during Network Fault Conditions" (IEEE ISIE,10 Bari Italy)

[9]. Glyn James and David Burely "Modern Engineering Mathematics" third edition, published Prentice Hall, ISBN: 01301831 99 9; pp 18-84

[10]. Narin G. Hongorani and Laszlo Gyugyi "Understanding FACTS Concept and Technology of Flexible AC Transmission Systems" published by IEEE Press. ISBN 0-7803-3455-8 